\title{
Evaluation of clinical, laboratory features and etiology of patients with febrile convulsion
}

\section{Suheyla Gumus ${ }^{1}$, Aslinur Ozkaya-Parlakay ${ }^{2}$, Alev Guven ${ }^{2}$, Belgin Gulhan ${ }^{3}$, Saliha Kanik-Yuksek ${ }^{2}$, Hasan Tezer ${ }^{2}$}

*1 Health Sciences University Ankara Hematology Oncology Children's Training and Research Hospital

*2 Health Sciences University Ankara Hematology Oncology Children's Training and Research Hospital,Pediatric Infectious Diseases Department

*3 Health Sciences University Ankara Hematology Oncology Children's Training and Research Hospital, Pediatric Neurology Department

Febrile seizures are the most common type Results: A case group consisting of two of benign seizures in childhood. Febrile seizures occurs in $\% 2$ to $\% 5$ of all children. Febrile seizures have been defined as a seizure, between 3 months and 5 years of age; associated with fever, but without evidence of central nervous system infection, acute electrolyte impairment, intoxication and history of afebrile seizure. The peak age of onset is 18 months and is more common in boys than in girls. The cause of a febrile seizure is unknown, but it is thought that to be multiple factors play a role in the pathogenesis. Many studies have examined the relationship between laboratory parameters such as iron deficiency anemia, mean platelet volume, sodium level with febrile seizures. In this study, it was planned to examine clinical characteristics and laboratory parameters of patients with febrile seizures between 6 months and 6 years of age and to compare findings with control group consisting of healthy children. Methods: This study was carried out in the Health Sciences University Ankara Child Health and Diseases Hematology Oncology Health Practice and Research Center Department of Neurology and Emergency Clinic with the patients who are diagnosed with febrile seizure and healthy children between 6 months and 6 years of age

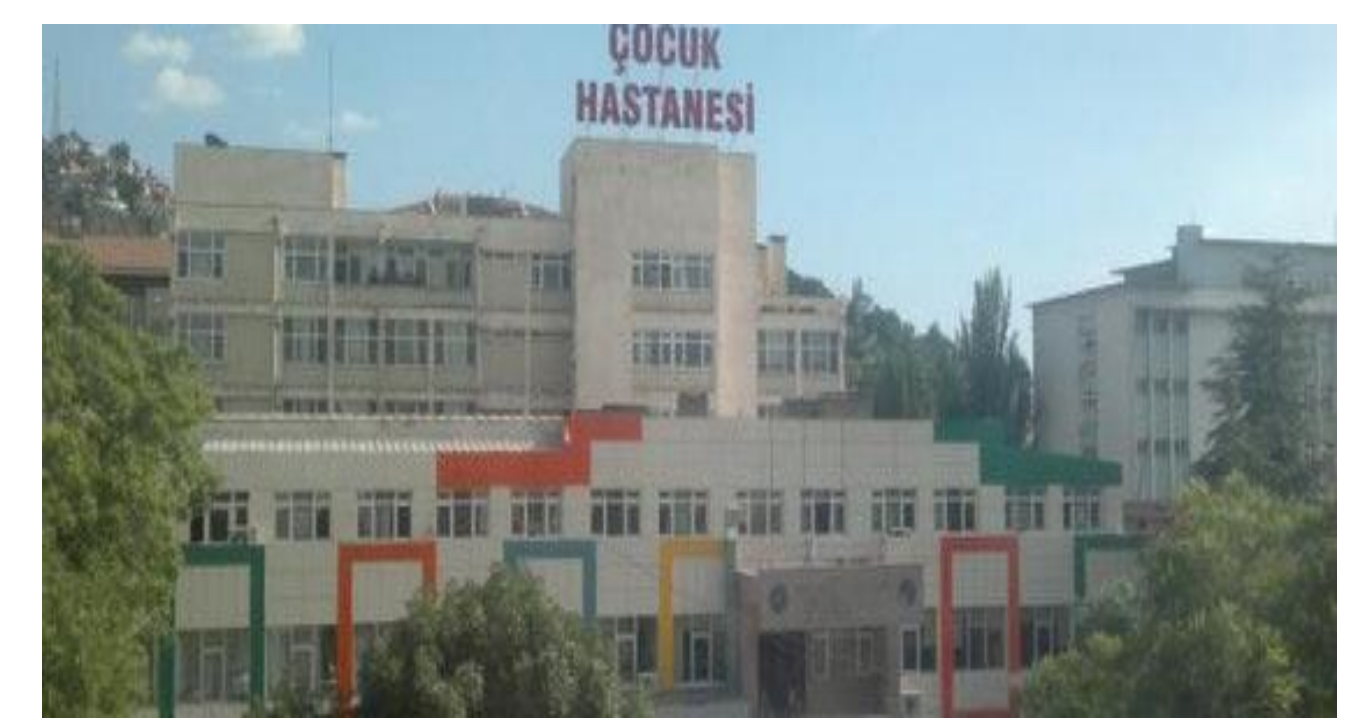
hundred thirty-three patients with febrile seizures and a control group consisting of two hundred thirty-three healthy children were included in our study. $54,5 \%$ of the patients were male, $45,5 \%$ were female. The mean age of the patients in the case group was 20,0 $\pm 11,2$ months and the mean age of the patients in the control group was $24,5 \pm$ 14,4 months. $78,1 \%$ of the patients were found to have simple febrile seizure and $21,9 \%$ of patients were found to have complex febrile seizure. $81,3 \%$ of the patients were found to have upper respiratory tract infection, $4 \%$ of the patients had lower respiratory tract infection, $9,4 \%$ of the patients had acute gastroenteritis, $4,5 \%$ of the patients had urinary tract infection and $0,9 \%$ of the patients had varicella infection. White blood cell count, neutrophil and creatinine values of the patients in the case the control group $(p<0,001, p<0,001, p=$ $0,002)$ and hemoglobin $(p<0,001)$, red blood cell count $(p=0,031)$, red cell distribution width, platelet $(<0.001)$, mean platelet volume $(p=0.028)$, lymphocyte, sodium, potassium $(p<0.001)$, AST $(p=0.01)$ and urea $(p=$ 0.001 ) values were significantly lower than the control group. According to seizure types in the case group, hemoglobin and urea values of the complex febrile seizure group were significantly lower than the simple febrile seizure group $(p=0.003, p=0.008$, respectively). There was a statistically significant difference between the types of seizures and the mean age of the patients who were followed up for febrile seizure $(p=$ seizure group was higher than the complex febrile seizure group. group were significantly higher than those of $0,003)$; the mean age of the simple febrile
When the patients in the case and contro groups were evaluated in terms of iron deficiency anemia, the iron deficiency anemia was found to be statistically higher in the case group than the control group $(p<0,001)$.

There was no significant difference between seizure type and iron deficiency anemia. Discussion: Iron is involved in the structure and synthesis of many enzymes and neurotransmitters in central nervous system, the neuronal excitability increases as the tissue iron falls below a certain level and since hemoglobin are molecules that carry oxygen, it is thought that in the case of anemia, the risk of seizures due to tissue hypoxia also increases.

Table 1. Laboratory parameters of control and patient group

\begin{tabular}{|c|c|c|c|c|c|}
\hline & & & & & $\mathbf{p}^{*}$ \\
\hline & Mean & $\pm \mathrm{SD}$ & Mean & $\pm \mathrm{SD}$ & \\
\hline WBC & 9476,8 & 2622,5 & 11906,4 & 5580,2 & ,000 \\
\hline $\mathrm{Hb}$ & 12,1 & 0,9 & 11,8 & 1,1 & ,000 \\
\hline KK & 4,8 & 0,4 & 4,8 & 0,4 & ,031 \\
\hline MCV & 74,3 & 6,5 & 74,3 & 5,5 & ,976 \\
\hline RDW & 14,9 & 1,6 & 14,3 & 2,0 & ,000 \\
\hline PLT & 352218,9 & 88260,2 & 305085,8 & 100885,4 & ,000 \\
\hline MPV & 7,7 & 0,8 & 7,5 & 0,7 & ,028 \\
\hline neutrophil & 3195,7 & 1665,6 & 7169,6 & 4739,4 & ,000 \\
\hline lymphocyte & 5282,3 & 1886,7 & 3395,0 & 1982,2 & ,000 \\
\hline $\mathrm{Na}$ & 137,0 & 2,3 & 134,3 & 2,6 & ,000 \\
\hline $\mathbf{K}$ & 4,5 & 0,4 & 4,2 & 0,4 & ,000 \\
\hline AST & 42,8 & 9,7 & 41,5 & 12,3 & ,010 \\
\hline ALT & 19,1 & 8,9 & 18,8 & 12,0 & ,305 \\
\hline BUN & 24,0 & 7,5 & 21,6 & 6,8 & ,001 \\
\hline creatinine & 0,4 & 0,1 & 0,5 & 0,1 & ,002 \\
\hline CRP & 0,2 & 0,1 & 2,0 & 2,5 &, 000 \\
\hline ferritin & 19,7 & 14,4 & 23,3 & 20,5 & ,509 \\
\hline
\end{tabular}

Conclusion: Recently, studies have been carried out that indicate the importance of mean platelet volume in acute vascular events and inflammatory processes. The iron deficiency anemia in the case group was found to be higher than the control group and mean platelet volume and sodium values of the case group were found lower than the control group. There is a need for more extensive prospective controlled studies in this area. 